\title{
Das Nerven-Kostüm
}

\section{Eine Alltagsmetapher $\nabla$}

Ich weiß nicht, ob Sie das kennen; aber mir passiert das manchmal: Da geht mir ein Wort einfach nicht mehr aus dem Kopf. Meistens ist es ein Wort, das ich seit vielen Jahren benutze, ohne großartig darüber nachzudenken.

Doch plötzlich hakt es sich irgendwo fest, von einer Sekunde auf die an-
Ein Wort geht nicht mehr aus dem Kopf.
Denn hören Sie bitte einmal genau hin: Da klingt doch bei der Verwendung von Nervenkostüm eine gewisse Distanz oder sogar Herablassung gegenüber dem Gemeinten an. Ja, ich glaube, Nervenkostüm ist eine im Grunde ironische Wortfügung. Sie versetzt den Sprecher in die Lage, über etwas zu reden, über das er eigentlich nicht reden möchte, weil es zu peinlich oder zu intim ist. Und wem, meine Damen Selbstverständlichkeit. Ja schlimmer noch: es wird zum Problem. Ich muss innehalten; und ich weiß: Ich werde so lange nicht in Ruhe weiterleben, wie ich diesem Wort nicht wenigstens ein Stück nach- oder entgegengegangen bin.

Eines dieser Worte ist „Nervenkostüm“. Sie kennen es alle. Es ist kein besonders auffälliges Wort. Es ist leidlich verbreitet; man benutzt es gelegentlich, doch eher beiläufig und ohne sich allzu viel dabei zu denken. Dabei ist es kein Begriff, sondern immerhin eine Metapher, also ein sprachliches Bild, in dem Bestandteile verschiedener Bereiche zu einer Vorstellung verschmolzen sind.

Ich frage mich also zunächst, wie mag dieses Wort Nervenkostüm wohl entstanden sein? Haben die Humanwissenschaftler es geprägt, als sie vor ein paar 100 Jahren feststellten, dass der menschliche Körper von einer Art Geflecht aus Nervensträngen durchzogen und dabei gewissermaßen innerlich umhüllt ist? Schaut man sich entsprechende Darstellungen an, könnte man diesen Wortursprung vermuten. Tatsächlich bilden die Nerven ja so etwas wie ein Kleid, wenngleich es natürlich unter der Haut getragen wird.

Nun glaube ich allerdings weniger an eine solche Geschichte der Wortentstehung. Ich vermute, das Wort ist entschieden jünger, vielleicht stammt es aus dem späten 19., vielleicht auch erst aus dem frühen 20. Jahrhundert. Der früheste Nachweis, den ich bislang habe finden können, stammt aus einem 1942 verfassten Frontbericht eines Wehrmachtsangehörigen. Und vielleicht ist gerade dieser Fund kein Zufall. Mein Sprachgefühl sagt mir nämlich, dass das Wort Nervenkostüm, wenn es nicht sogar im militärischen Kontext entstanden ist, dort zumindest starke Verbreitung gefunden hat. und Herren, sollte das Sprechen über seine nervliche Verfassung peinlicher sein als dem Soldaten, der ja quasi von Berufs wegen dazu verpflichtet ist, starke oder besser überhaupt keine Nerven zu haben. Stellen Sie sich also bitte versuchsweise den schneidigen Oberleutnant aus dem Jahr 1914 vor. Jahrelang hat er über alles und jedes im Kasinotonfall daherschwadroniert, aber jetzt hat ihn angesichts der Materialschlachten an der Westfront die nackte Angst erfasst, sodass er vorübergehend in einer entsprechenden Einrichtung behandelt werden muss. Wieder entlassen, wird er den Teufel tun und offen über seine angegriffene Psyche reden. Stattdessen wird er nach Metaphern greifen, die es ihm ermöglichen, sich zumindest sprechend von seiner Schwäche zu distanzieren. Also wird er vielleicht sagen (denken Sie sich jetzt ein Monokel in meinem Auge): „Na, da hat der werte Herr Feind mir doch unfreundlicherweise ein paar Löcher ins Nervenkostüm geschossen. Musste kurze Auszeit nehmen und das flicken lassen."

Ich bin mir ziemlich sicher, dass es genau diese ironische Distanzierung ist, die das lassen. Denn ganz ehrlich: Wer von uns Menschen freut sich schon ungeschmälert an dem Umstand, dass wir ein lebenswichtiges Sensorium besitzen, dessen Funktionen und Parameter sich so wenig kontrollieren lassen. Unsere Hände sind unsere geliebten Werkzeuge, jedenfalls solange sie stark sind und wir sie steuern können. Unsere Nerven hingegen scheinen uns nie so recht zu gehören. Wir sind zwar auf ihre Leistungen angewiesen, um überleben zu können, doch gleichzeitig sind wir ihnen auch ausgeliefert. In den einschlägigen Redewendungen findet sich aufgehoben, was uns hier so alles pasWort Nervenkostüm hat Karriere machen

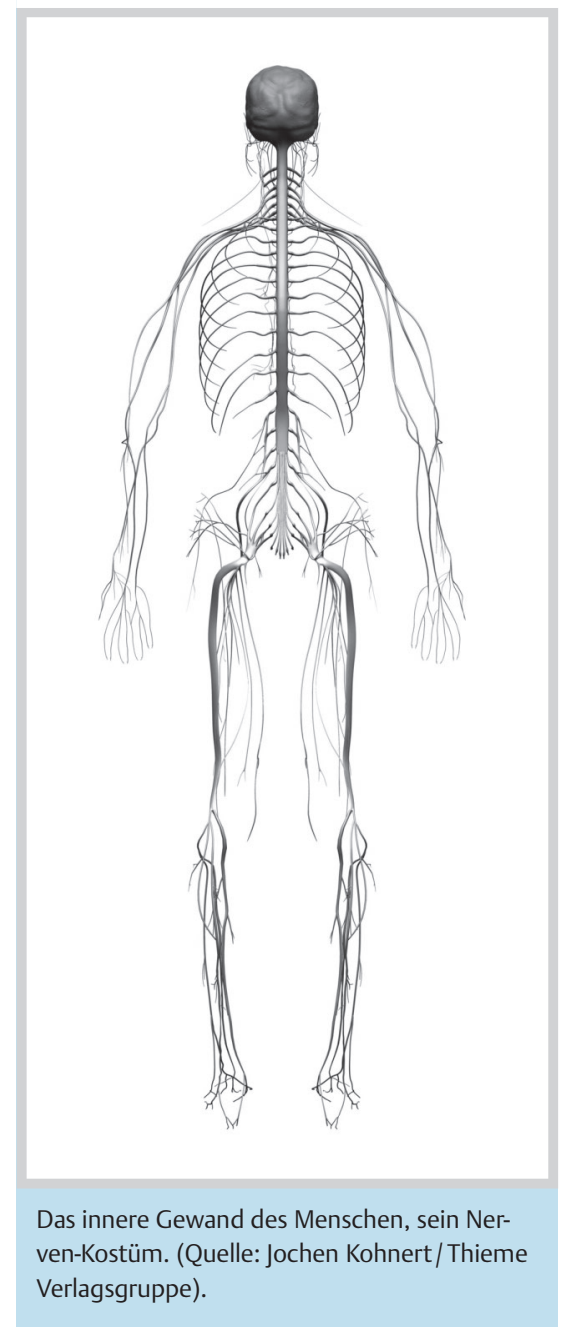

sieren kann: Unsere Nerven können strapaziert werden, gespannt sein und dünn werden, sie können flattern und reißen, wir können sie sogar vollständig verlieren. Und das alles geschieht ohne unser Zutun, zwar innerhalb unserer Körpers, aber außerhalb unseres Willens. Wir haben, um eine andere Redewendung zu zitieren, unsere Nerven einfach nicht im Griff. Ja, schlimmer noch: Unsere Nerven können sich sogar selbstständig machen, um uns Streiche zu spielen.

Ich denke, dieser ganze Umstand ist in der Metapher Nervenkostüm aufgehoben. Wir tragen unsere Nerven wie ein Kostüm, d.h.: Sie kleiden uns, sie geben uns unseren Charakter und unsere innere Gestalt, doch zugleich empfinden wir sie als nicht ganz unser eigen - eben als ein Kostüm, also als etwas, das Kleid, aber auch Verkleidung sein kann, etwas Bergendes, 
aber auch etwas, das uns gegen unseren Willen bestimmt.

Durch die Metapher Nervenkostüm nun halten wir uns unsere Nerven ein wenig vom Leib, indem wir eine leichte ironische Distanz dazu schaffen. Wer sagt „Ich habe schlechte Nerven“, der steht ganz anders zu dem Problem als der, der sagt „Mein Nervenkostüm bekommt Risse“. Mit der ironischen Metapher wehren wir uns dagegen, mit unseren Nerven identisch gesetzt zu werden. Obwohl sie in Wahrheit sogar unter unserer Haut liegen, halten wir sie auf Abstand. Das heißt natürlich, wir versuchen es! Die Metapher dient als Beschwörung. Sie artikuliert den Wunsch, dass wir unseren Nerven nicht völlig ausgeliefert sein mögen. Ja, sie formuliert sogar die Hoffnung, es könnte möglich sein, die Nerven zu wechseln - ebenso wie man ein Kostüm wechselt.

\section{Nerven nach der Mode: 1970}

Soweit die erste Phase meiner Überlegungen zum Wort Nervenkostüm. Doch wie das so kommt, wenn man einem Wort nachgeht: Es lockt einen auf immer neue Wege und stimuliert weitere Spekulationen. Und da ich der festen Überzeugung bin, dass selbst in den schlichtesten Worten unserer Sprache, insbesondere aber in den Metaphern, große Gedankengebäude höchst verdichtet gespeichert sind, hat mich die Beschäftigung mit dem Wort Nervenkostüm zu einer weiteren Frage inspiriert. Wenn nämlich, so frage ich mich, das Nachdenken über den Charakter der Nerven zu der Vorstellung vom Kostüm geführt hat, könnte es dann nicht möglich sein, dass das Nervenkostüm wie alle anderen Kostüme der Mode unterworfen ist?

Ich weiß, die Frage mag sich ein wenig kühn anhören. Daher will ich gleich mit einem konkreten Fall beginnen, besser gesagt, mit dem historischen Fall, auf den ich bei meinen Überlegungen sofort und mit Notwendigkeit gestoßen bin. Ich selbst bin Jahrgang 1956, bin also der 68er-Generation ein wenig nachgeboren und ihren Protagonisten später auch eine Zeitlang hinterhergelaufen, notgedrungen; jemand anders zum Hinterherlaufen bot sich mir jedenfalls nicht an. Wer von Ihnen das kann, der erinnere sich doch jetzt bitte an die späten 60er- und die frühen 70er-Jahre. Es war nicht zuletzt die Zeit eines vehement ausgetragenen Generationskonflikts. Unversöhnlich standen Eltern und Kinder einander gegenüber; und insbesondere die Jungen taten alles, um nicht mit ihren Eltern verwechselt zu werden. Sie trugen ganz andere Kleidung und ganz andere Frisuren, sie hatten ganz andere Überzeugungen, sie praktizierten ganz andere Lebensformen, und - sie trugen ein ganz anderes Nervenkostüm. Genauer gesagt: Es gehörte zum zeittypischen Dresscode, ein Nervenkostüm zu tragen, das etwa so aussah wie die indischen Gewänder der
Ließ ein stählernes Nervenkostüm den Krieg ertragen?
Zweck es wiederum sein sollte, die Sensibilität ihrer Konsumenten noch weiter zu verfeinern und, wie es damals hieß, ihr Bewusstsein zu erweitern.

\section{Nerven nach der Mode: 1940}

Ich denke nun, dieses modische Nervenkostüm der Jahre um 1970 kann nicht ganz verstanden werden ohne eine andere „Nervenmode“, zu der es einen dezidierten Gegenentwurf markieren wollte. Deren Träger aber waHippies. Es war abwechslungsreich farbig, aber vor allem sanft und dünn, fast durchsichtig, weil sehr sehr fein gewoben. Wenn es berührt wurde, ja wenn man es nur anhauchte, zitterte es und warf lange, feine Falten. Harten Kontakt ertrug es nicht, dann riss es.

Oder anders gesagt: Es gehörte in den Jahren um 1970 zum bewusstseins- oder empfindungsgeschichtlich aktuellen Habitus, ein äußerst sensibler Mensch zu sein. So galt es als Muss, eine starke Sympathie zu anderen Kreaturen zu pflegen, wörtlich übersetzt: ein Mitleiden, selbst wenn dessen Objekte ganz anderswo auf der Welt lebten und man von ihrem Leid nur durch Flugblätter erfahren hatte. Auch im persönlichen Umgang miteinander galten die Aufmerksamkeit für schwache oder versteckte Signale und eine dauernde Bereitschaft zur Thematisierung des Emotionalen als Pflichtübungen. Allgegenwärtig war die Aufforderung, sich selbst und andere zu sensibilisieren: sei es für Politisches, sei es für die Gefährdung der Natur, für die Sexualität oder für unterschwellige Empfindungswelten.

An die Dehors und Accessoires, die zu diesem Nervenkostüm gehörten, erinnert sich heute mancher mit Wehmut, mancher aber auch mit Grausen. Räucherstäbchen und indische Sitra-Musik. Lange Haare bei Männern, auch als Zeichen einer eher femininen Gefühlsverfassung. Die John-Lennon-Brillen, die jedem Gesicht etwas Kindlich-Fragend-Naives und damit auch etwas Empfindliches gaben. Weiche Matratzenlager als Protest gegen die zwanghafte bürgerliche Wohnkultur. Surrealistische Gemälde von Salvatore Dali und natürlich das ganze Spektrum von Drogen, deren vornehmlichster ren die Eltern von 1970, zugleich die Hitlerjungen und BDM-Mädchen der 30erJahre. Die aber waren in einem bewusst asensiblen, ja in einem animalischen Geist erzogen worden. Ich zitiere die berühmte Stelle aus Hermann Rauschnings Aufzeichnungen seiner Gespräche mit Hitler (natürlich nicht ohne den Hinweis, dass der Wortlaut historisch nicht verbrieft ist. Man kann ja nicht vorsichtig genug mit seinen Quellen umgehen).

Hitler (nach Rauschning, S. 237): „Meine Pädagogik ist hart. Das Schwache muss weggehämmert werden. In meinen Ordensburgen wird eine Jugend heranwachsen, vor der sich die Welt erschrecken wird. Eine gewalttätige, herrische, unerschrockene, grausame Jugend will ich. [...] Schmerzen muss sie ertragen. Es darf nichts Schwaches und Zärtliches an ihr sein. Das freie, herrliche Raubtier muss erst wieder aus ihren Augen blitzen. [...] Ich will keine intellektuelle Erziehung. Mit Wissen verderbe ich mir die Jugend. [...] Aber Beherrschung müssen sie lernen. Sie sollen mir in den schwierigsten Proben die Todesfurcht besiegen lernen.“

Die Todesfurcht besiegen! Kann es eine stärkere Leistung der Nerven geben? Egal, wie authentisch diese Sätze nun sein mögen; sie vermitteln doch sehr gut die emotionalen Ideale der NS-Zeit, wie sie damals von Tausenden sog. Volkserziehern ähnlich formuliert und in der pädagogischen Alltagspraxis umgesetzt wurden. Und was die Nachkriegsgeneration dann in den 50er- und 60er-Jahren von ihren Eltern und über ihre Eltern erfuhr, das deckte sich auch scheinbar weitgehend mit diesen Idealen. So wurden Geschichten aus dem Krieg eher selten als Geschichten des Leidens und der Verzweiflung vermittelt, viel häufiger aber als Geschichten des Heldentums, des nervenstarken Aushaltens und des klaglosen Ertragens. Die Söhne und Töchter der Vor- 
kriegs- und Kriegsmenschen mussten glauben, es sei tatsächlich eine existenzielle Dickfelligkeit, oder anders gesagt: ein stählernes Nervenkostüm, das ihre Eltern in die Lage versetzt habe, die Katastrophe des Krieges nicht nur auszuhalten, sondern sie nach 1945 sogar umgehend zu vergessen.

Selten genug wurde diese Vorstellung hinterfragt. Komplexere Überlegungen wollte um 1970 kein 20-Jähriger anstellen. Dabei waren ja die Verdrängung des Kriegsgeschehens und die unsensible Fixierung aufs Ökonomische in den 50erJahren vielleicht das Resultat einer kollektiven Traumatisierung. Vielleicht hatte man ja eine ganze Empfindungsgeneration nur mit brutaler Gewalt in jenes stählerne Nervenkostüm gezwängt, freilich mit dem Resultat, dass sie jetzt nicht wieder herauskonnte, ohne ihre erlernten Ich-Bilder zu verraten. Was dazu führte, dass sie wie in einem emotionalen Panzer lebte, unglücklich und autistisch.

Aber wie gesagt: Der Generationenkonflikt um 1970 war nun einmal weniger von gegenseitigem Verständnis als vom Willen der Jüngeren geprägt, um jeden Preis anders als die Alten zu sein. So kam es dazu, dass der von oben verordneten Nervenstärke der gewesenen Hitlerjugend eine von unten verordnete Nervenschwäche der Hippie- und Protestjugend entgegengesetzt wurde. Dieser Zwang zu einer Empfindsamkeit, die von einfacheren Gemütern auch gerne als Empfindlichkeit ausgelebt wird, hat sich, in gewissen Schichten und Kreisen, noch lange, ja teilweise bis heute, erhalten. Ich nenne hier die besonders in der Politik gepflegte Betroffenheitskultur, deren größte Leistung die Durchsetzung der Political Correctness im öffentlichen Sprechen gewesen ist. Und ich erinnere daran, dass das Empfindungsklima an unseren Schulen noch weitgehend von der Empfindungskultur der 70er-Jahre geprägt ist, was dazu führt, dass viele junge Menschen den Übergang ins Berufsleben als nervenzerreißend empfinden, beziehungsweise empfinden müssen.

\section{Entwurf zu einer Theorie der Nervenmoden \\ $\nabla$}

Dieses historische Beispiel eines NervenKostümwechsels, das ich selbst miterleben durfte oder musste, hat mich nun dazu verführt, zumindest den Entwurf einer übergreifenden Theorie und Geschichte der Nervenmoden zu skizzieren.
Ich kann Ihnen hier natürlich nur eine Vorschau auf dieses gewaltige Forschungsprojekt geben, das ich demnächst auch in andere Hände werde legen müssen, da es meine Möglichkeiten übersteigt.

Der historische Raum, den meine Geschichte der Nerven-Kostümmode umgreifen sollte, beginnt im 18. Jahrhundert und reicht bis zur Gegenwart. Ich gebe Ihnen eine kleine Übersicht über das Forschungsgebiet.

Denken Sie sich bitte zunächst den Menschen der Aufklärung. Er steht vor der gewaltigen Aufgabe, den christlichen Glauben als alleiniges Fundament des Weltverständnisses zu verlassen und stattdessen die menschliche Vernunft und die wissenschaftlichen Erkenntnismethoden als Mittel und Motor der Weltdeutung zu etablieren. Sie können sich vorstellen, welch starke Nerven man für dieses Unterfangen benötigt. Der Rationalismus fordert den Kopfmenschen, der sich unbeirrt seiner Aufgabe stellt und dabei versucht, alles Individuelle als Störfaktor auszuschalten. Hatte sich die Religiosität der vergangenen Jahrhunderte bei den allermeisten Menschen als Herzenssache oder als Seelenangelegenheit manifestiert, so sind jetzt Kopf und Verstand die Akteure des Zeitbewusstseins. Den Aufklärer denke man sich also als einen Menschen in einem streng geschnittenen Nervenkostüm, das alle individuellen Körper- und Geistesformen verbirgt.

Kein Wunder also, dass es, wenngleich unter anderen historischen Vorzeichen, auch damals schon eine Revolte gegen diese strenge Nervenmode gab. Die Nerven-Revolte der Jahre um 1970 habe ich nun noch aus der persönlichen Erinnerung beschreiben können; für die des 18 . Jahrhunderts aber muss ich auf ihren Niederschlag in der zeitgenössischen Literatur zurückgreifen. Im Zuge des Protestes gegen eine einseitige Betonung des Verstandes, ja als Träger der Gegenbewegung, entstand die literarische Strömung der Empfindsamkeit, deren wichtigste deutsche Vertreter die Autoren Klopstock und Lessing sind. Sinnlichkeit und Innerlichkeit, die in der frühen Phase der Aufklärung als Makel gegolten hatten, wurden jetzt als wichtige, ja als notwendige Bestandteile menschlichen Lebens wiederentdeckt. Das Theater leistete hier die Hauptarbeit. In den sog. Rührstücken dieser Epoche ging es ausdrücklich darum, das Publikum zu großen Gefühlen hinzu- reißen; dabei waren den populären Theaterautoren wie Iffland und Kotzebue auch die gröbsten Mittel recht. Von ihren Werken zieht sich eine ununterbrochene Linie bis zu den Samstagabendfilmen im Fernsehen, in denen jede Frage nach Sinn oder Glaubwürdigkeit des Plots von der einen Intention des Ganzen, den Zuschauer zu Tränen zu rühren, einfach weggeschwemmt wird.

$\mathrm{Zu}$ den wesentlich wertvolleren Dokumenten der Empfindsamkeit gehört neben den bürgerlichen Trauerspielen Lessings insbesondere Goethes „Leiden des jungen Werthers“. Wie kaum ein literarisches Werk vor ihm war der „Werther“ imstande, einer ganzen Empfindungsgeneration ihr Nervenkostüm zu entwerfen. Und das sogar im nichtmetaphorischen Sinne. Tatsächlich wurde Werthers Tracht, blaue Jacke und gelbe Weste, zu einem modischen Ensemble. Insbesondere aber wurde seine überaus sensible Art, Umwelt, Mitmenschen und sich selbst wahrzunehmen, zum viel kopierten Vorbild. Das soll so weit gegangen sein, dass sein Selbstmord aus Liebeskummer mehrfach kopiert wurde.

Mit dem Werther zieht eine Figur ins öffentliche Bewusstsein ein, die gegen alle Tugendregeln des aufgeklärten Bürgertums verstößt und an dieser Revolte zerbricht, dabei aber eine Frage aufwirft, die bis heute immer wieder gestellt wird: Es ist die Frage, ob es nicht ehrlicher und menschlicher ist, seinen individuellen Gefühlen zu leben, als sich den Regeln der Gesellschaft zu unterwerfen. Werther bezahlt seine Unfähigkeit, gegen seine Gefühle zu leben, sich gewissermaßen emotional zu immunisieren, mit dem Tod. Aber vor diesem Tod stehen alle Virtuosen und Schwergewichtsmeister der Selbstbeherrschung dann doch ein wenig ärmlich da. Werther ist einer der ganz großen Sensiblen, die gerade durch ihre Schwäche die Nervenstarken blamieren.

Der Konflikt zwischen den beiden Nervenmoden von Aufklärung auf der einen und Empfindsamkeit auf der anderen Seite wiederholt sich in Deutschland am Anfang des 19. Jahrhunderts, als sich, unter wieder anderen bewusstseinsgeschichtlichen Vorzeichen, die Vertreter der literarischen Romantik zu Wort melden, um einzuklagen, was beim allmählichen Erstarken der bürgerlichen Gesellschaft auf der Strecke zu bleiben droht. Diesmal geht es aber eher um den Verlust der Religiosität, oder besser gesagt: um den Ver- 
lust der Transzendenz im Alltag. Etwas verkürzt gesagt, ist das modische Nervenkostüm der Romantik das des Taugenichts und Schwärmers. Der Taugenichts erträgt es schon körperlich nicht, in die strengen Nützlichkeitskorsetts des Bürgertums gesperrt zu werden; der Schwärmer ist den Reizen seiner Umwelt, insbesondere dem Reiz der unberührten Natur, zu sehr ausgesetzt, als dass er ein gradliniges und zielorientiertes Leben führen könnte.

\section{Die Hochzeit der Nervenkunst: 1900 \\ $\nabla$}

Ich kann die Romantik aber nur kurz streifen, weil ich in der mir verbleibenden Zeit noch unbedingt eine absolute, ja die Hochzeit der Nervenmode schlechthin erwähnen muss. Es ist das die Zeit um die vorletzte Jahrhun-
Wiedergabe des Sinneseindrucks als höchste Stufe der Kunst. zurückzuschlagen, meistens mit der „Berliner Schnauze“.

Eben diese modernen Großstädte aber sind ausgangs des 19. Jahrhunderts auch der Geburtsort einer gerade entgegengesetzten Nervenmode. Tatsächlich ist die gesamte europäische Kunst dieser Zeit bestimmt von einer Kritik am realistisch-mathematischen Weltbild. Einen großen Anteil daran hatte die junge Psychologie, die zeigt, wie wenig rational Menschen agieren und wie sehr sie von ihren Trieben, von ihren unreflektierten, ja unkontrollierbaren Vorlieben und Abneigungen gesteuert werden. Sehr prägend ist auch der Empiriokritizismus des Physikers und Wissenschaftstheoretikers Ernst Mach, der darauf hinauslief, dass eine Trennung zwischen Welt und Ich nicht vollzogen werden kann, hat die bürgerliche Kultur mit der Technisierung und Industrialisierung des Alltags ihre raumgreifendste Leistung abgeliefert; durch Dampfmaschine, Motor, Elektrizität und Telefonie hat sich die Welt vollkommen verändert. Das Nützlichkeitsdenken der bürgerlichen Welt hat als seinen Ausdruck die Maschine gefunden, eine Maschine, in der die ganze Gesellschaft aufzugehen scheint. Dabei verlangt der Kontakt mit diesen gewaltigen Umwälzungen Nerven aus jenen schier unzerreißbaren Stahlseilen, die damals erstmals hergestellt werden können. Schon das bloße Leben in der pulsierenden Großstadt erfordert ein anderes Nervenkostüm als es die Landbevölkerung der vergangenen Jahrhunderte getragen hatte. So prägt sich dann auch in den großen Städten eine spezifisch urbane Mentalität aus, deren wichtigster Bestandteil die Resistenz gegen den Lärm, das Tempo und die dramatische Überfüllung des Lebensraumes ist. Prominentester Träger dieses neuen Nervenkostüms ist in Deutschland der Berliner, der sich einerseits durch nichts aus der Ruhe bringen lässt, andererseits aber immer bereit und in der Lage ist, Angriffe auf sein Nervenkostüm schnell und rabiat

\section{Literatur ist Nervensache} $\nabla$

Und damit abschließend kurz ein paar Worte zum eigentlichen Ziel meines ,Forschungsprojektes“ „Modegeschichte des Nervenkostüms“. Als Schriftsteller, und zumal als einer, der gerade an einem Buch über die Feigheit schreibt, geht es mir immer auch darum, die komplexe Psyche meiner Figuren als Resultat überindividueller Kräfte und Strömungen zu zeigen. Literarische Figuren, die auf nichts als sich selbst weisen, sind uninteressant, ja irrelevant. Viel lohnenswerter ist es zu zeigen, von wo sich die Protagonisten ihr Nervenkostüm besorgt haben, ob es nur geliehen oder ihnen sogar aufgezwungen wurde. Es ist die Aufgabe der Literatur, das Allgemeine noch im scheinbar Allerprivatesten, etwa in der nervlichen Verfassung ihrer Helden kenntlich zu machen. Literatur will ihre Helden nicht heilen wie die Medizin ihre Patienten, sie entwirft vielmehr in speziellen Krankengeschichten den $\mathrm{Zu}$ stand des großen Ganzen. Also zeigt sie, ob eine dünne Haut nicht vielleicht ein modisches Kostüm ist oder ein dickes Fell bloß eine Zwangsjacke.

Ich danke abschließend sehr herzlich für die Gelegenheit, mit meiner literarischen Variante des Nervenkostüms in dieser medizinisch-wissenschaftlichen Welt kurz aufscheinen zu dürfen. Vielleicht konnte ich ein wenig Neugier wecken auf meine Welt der Literatur. Und die könnte sich lohnen. Denn die Schriftsteller lügen - aber ihre Lügen sind oft einflussreicher als die Wahrheiten der Wissenschaft.

Ein kleines Beispiel? Bitte! Zu dem verbreiteten Satz „Der Indianer kennt keinen Schmerz“, mit denen zu Zeiten der groberen Nervenkostüme hierzulande die Kinder erzogen wurden, gibt es erstaunlicherweise kein englisches Pendant. Tatsächlich war es nämlich ein deutscher Schriftsteller, der einmal mutmaßte, „die Nerven des Roten“ könnten „weniger empfindlich als diejenigen des Weißen“ sein, und damit zum Volkserzieher wurde. Sein Name ist Karl May. 


\section{Zur Person}

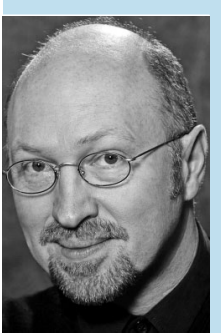

Burkhard Spinnen, 1956 in Mönchengladbach geboren; Studium der Germanistik, 1989 Promotion über Kurze Prosa; bis 1995 wissenschaftlicher Assistent am Germanistischen Institut der Universität Münster; seitdem freier Schriftsteller. 1998-2000 Vertreter einer Professur für Literarische Ästhetik am Deutschen Literaturinstitut Leipzig. 2000-2006 Juror beim Ingeborg-Bachmann-Preis in Klagenfurt, seit 2008 Vorsitzender der Jury. Mitglied des deutschen PEN und der Akademie der Wissenschaften und Künste NRW.
Veröffentlichungen: Erzählungsbände, Romane, Kurzprosabände, daneben wissenschaftliche und essayistische Texte, insbesondere zur Literatur, zur zeitgenössischen Medien- und Sprachkultur und zum Verhältnis von Kultur und Wirtschaft; Rezensionen, Kommentare und Glossen für Hörfunk, Zeitungen und Zeitschriften zu literarischen, politischen und sozialen Themen.

Auszeichnungen u. a.: Aspekte-Literaturpreis des ZDF (1991), Literaturpreis der Konrad-Adenauer Stiftung (1999), Deutscher Hörbuchpreis (2008), Rheinischer Literaturpreis (2009)
Korrespondenzadresse

Dr. Burkhard Spinnen

Mondstraße 137

48155 Münster

bspinnen@aol.com

Bibliografie

Dol http://dx.doi.org/

10.1055/s-0031-1276925

Klin Neurophysiol 2011; 42: 194-198

(c) Georg Thieme Verlag KG

Stuttgart · New York

ISSN 1434-0275 